\title{
Web Ontology Language (OWL) and Semantic Web
}

\author{
Goutam Kumar Saha
}

Ontology is a sub-field of Philosophy. It is the study of the nature of existence and a branch of metaphysics concerned with identifying the kinds of things that actually exists and how to describe them. It describes formally a domain of discourse. Ontology is used to capture knowledge about some domain of interest and to describe the concepts in the domain and also to express the relationships that hold between those concepts. Ontology consists of finite list of terms (or important concepts) and the relationships among the terms (or Classes of Objects). Relationships typically include hierarchies of classes. It is an explicit formal specification of conceptualization and the science of describing the kind of entities in the world and how they are related (W3C). Web Ontology Language (OWL) is a language for defining and instantiating web ontologies (a W3C Recommendation). OWL ontology includes description of classes, properties and their instances. OWL is used to explicitly represent the meaning of terms in vocabularies and the relationships between those terms. Such representation of terms and their interrelationships is called ontology. OWL has facilities for expressing meaning and semantics and the ability to represent machine interpretable content on the Web. OWL is designed for use by applications that need to process the content of information instead of just presenting information to humans. This is used for knowledge representation and also is useful to derive logical consequences from OWL formal semantics.

\section{OWL \& Semantic Web (SW)}

The Semantic Web (a revolutionary vision of Tim Berners-Lee) is nothing but the second-generation Web. It weaves together a network of information that allows more efficiency, greater knowledge sharing, and ease of use. Ontologies are the key to this interoperability because they determine the language that software agents need to communicate with each other and humans need to communicate with the agents. Semantic Web will enable automatic collection and correlation of various parts of information about an object, available at various different web resources. Semantic Web will save our valuable time we spend on navigating from one web resource to another in order to obtain meaningful information on a particular object. We would be happy then on finding out for example, our old friend's complete information on giving partial hints on the fly without the need of our manual visiting various related web pages! The Semantic Web is a vision of W3C led by Tim Berners-Lee for the future of the web in which information is given explicit meaning, making it easier for machines to automatically process and integrate information available on the Web. Semantic Web is a web of data and it provides a common framework that allows data to be shared and reused across application, enterprise and community boundaries. The Semantic Web is 
envisaged as a place where data can be shared and processed by automated tools as well as by people. The key lies in the automation and integration of processes through machine-readable languages. In order to leverage and link the vast amounts of information available on the Web, software agents must be able to comprehend the information, i.e., the data must be written in machinereadable semantics.

The Semantic Web is being built on XML's ability to define customized tagging schemes and on RDF that is a flexible approach to represent data and on ontology language (OWL) at the first level above RDF that can formally describes the meaning of terminology used in Web documents. In order for computers to be more helpful, the Semantic Web augments the current Web with formalized knowledge and data that can be processed by computers. In order to enable to search and process information such as airline flights, software programs or software agents need information that has been modeled in a coherent manner. Ontology models all the entities and relationships in a domain.

\section{OWL Sub-languages}

OWL provides three increasingly expressive sub-languages designed for use by specific communities of implementers and users:-

(i) OWL Lite (is least expressive, suitable for simple class hierarchy and simple constraints and useful for quick migration path for thesauri and other taxonomies),

(ii) OWL DL (is more expressive, retains Computational Completeness that is, all conclusions are guaranteed to be computable and has Decidability that is, all computations will finish in finite time, and is based on Description Logic),

(iii) OWL Full (is most expressive and has syntactically freedom of RDF and has no computational guarantees but allows an ontology to augment the meaning of the pre-defined (RDF or OWL) vocabulary and is not suitable for auto-reasoning).

\section{OWL Ontology Components}

An OWL Ontology consists of the following components:-

(i) Individuals or Instances or Objects (No Unique Name Assumption UNA)

(ii) Properties or Relations (e.g. Binary relations- hasChild)

(iii) Classes or Concepts (Subclasses are subsumed by their superclasses. Classes are described using formal or mathematical descriptions stating requirements for membership of the class). 


\section{Properties Representation}

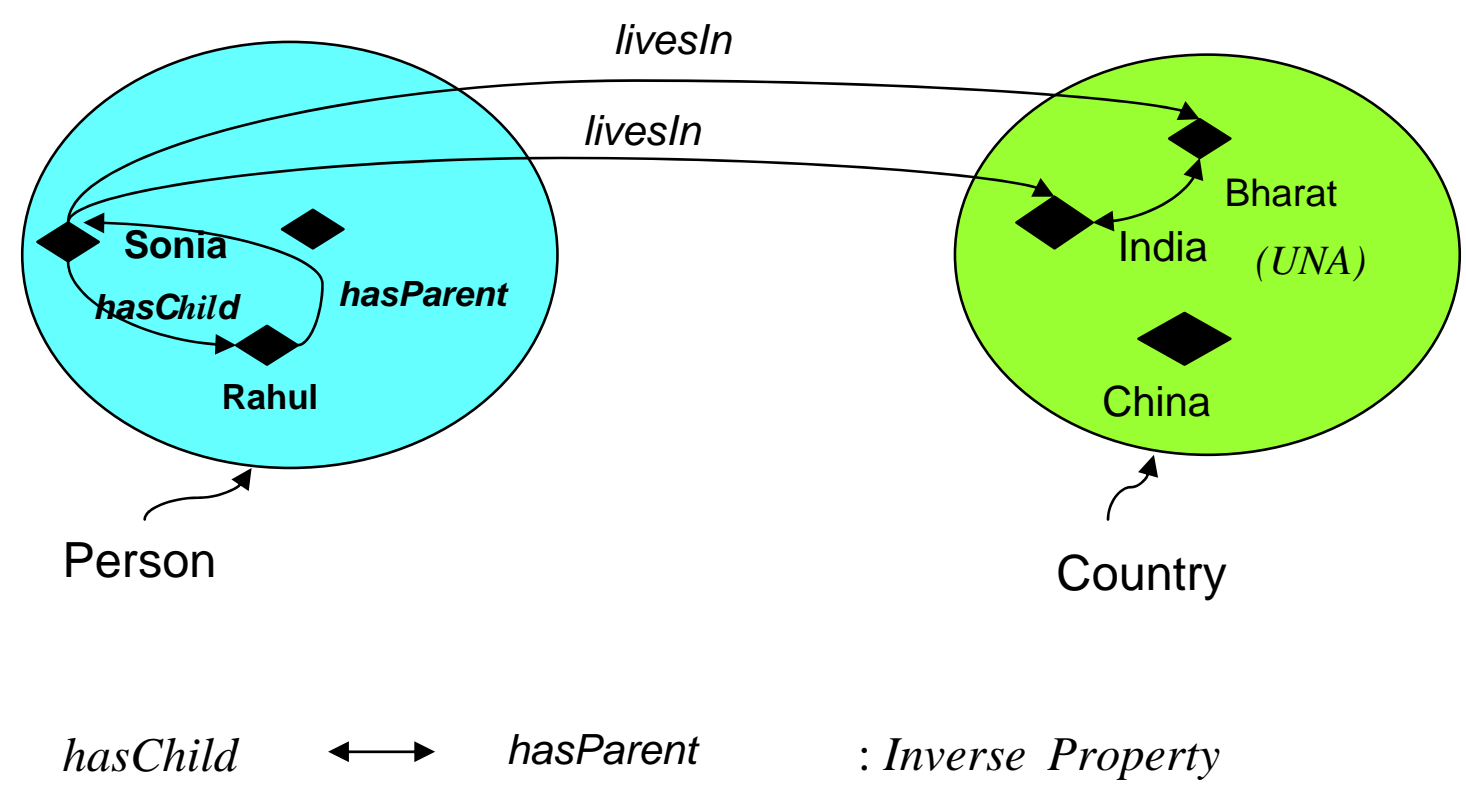

Figure-1. Properties Representation

In the above figure-1, both Sonia and Rahul are the individuals of the Person Class. India and Bharat have been indicated as the same instances of the Country Class. Sonia has child named Rahul and Rahul has the parent called Sonia. The relations "hasChild" and "hasParent" are inverse to each other.

\section{RDF or Resource Description Framework}

RDF is a framework for describing web resources (identified by Uniform Resource Identifier or URIS) such as homepage, title, author, content and copyright information of a web page etc. RDF is a data model for objects ("resources") and relations between them. RDF provides a simple semantics for this data model, and these data models can be represented in XML syntax. RDF is designed to be read and understood by computers but it is not meant for displaying to human being. RDF describes resources with properties and property values. A resource is anything that can have a URI, such as http://www.kolkataabcd.in/NLE.

A property is also a resource that has a name, such as "author" or "homepage." A property value can also be another resource. RDF is a basic ontology language and a graphical language used for representing information about 
resources on the web. Resources are described in terms of properties and property values using RDF statements. Statements are represented as triples, consisting of a subject, predicate and object $[\mathrm{S}, \mathrm{P}, \mathrm{O}]$. The subject of one statement may be the object of another statement and that is how resources can be merged together. A set of linked statements (triples) forms an RDF Graph.

\section{$\underline{\text { RDF Code }}$}
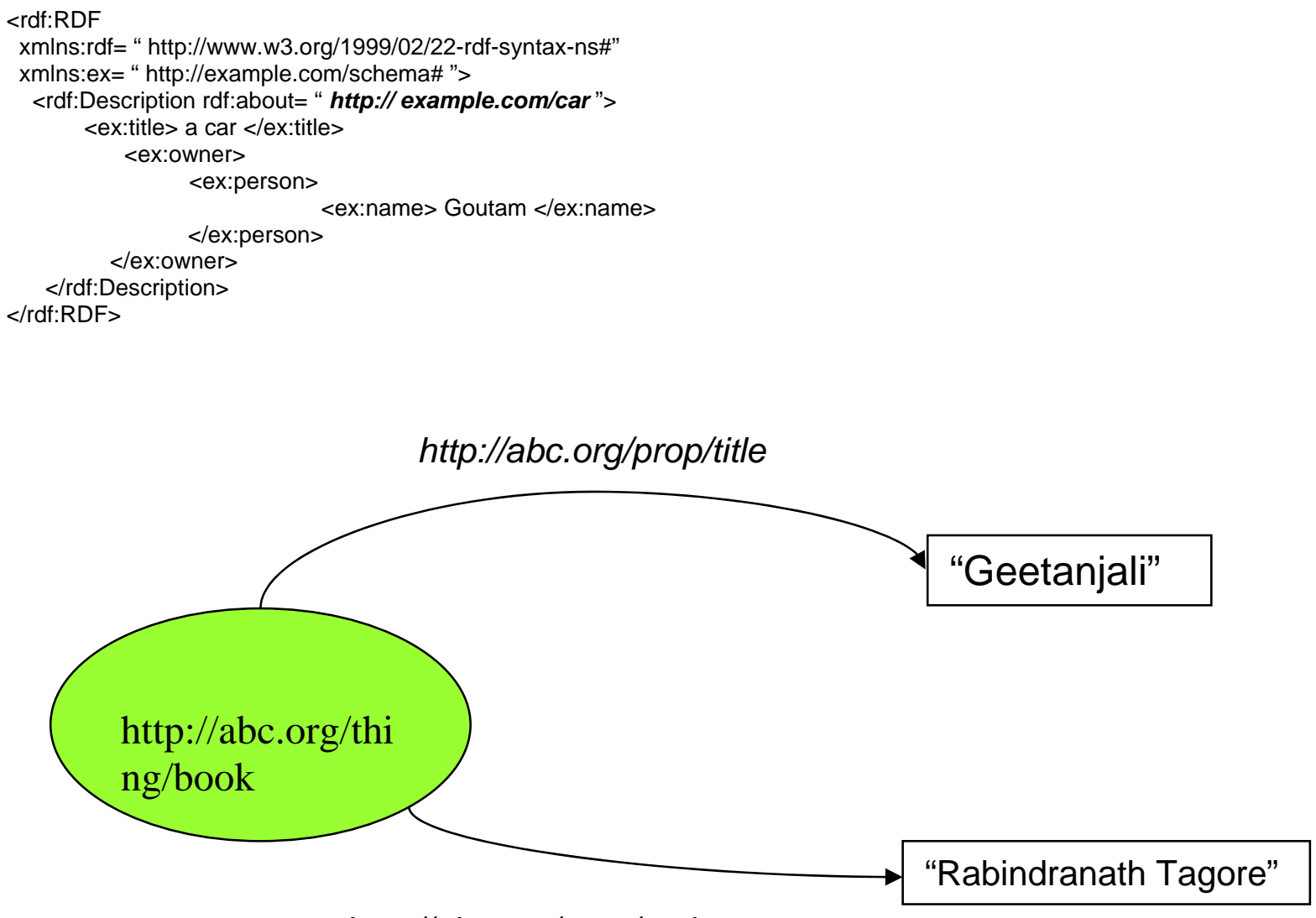

http://abc.org/prop/author

Subject

Values

Properties

Figure-2. RDF Statement Triplet

\section{RDFS (RDF Schema)}

RDFS is an extension to RDF that provides the framework to describe application- specific classes and properties and thus allows resources to be defined as instances of classes, and subclasses of classes. RDFS allows 
properties to be defined as sub properties of other properties and it enriches the descriptions of what we already have.

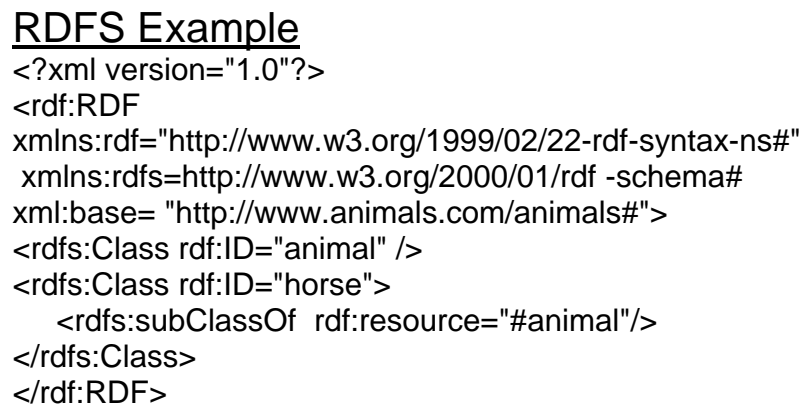

\section{RDFS -Describing Classes}

To describe classes we can use built in RDF Schema resources such as,

- rdfs:Class

- rdfs:subClassof

These are used in conjunction with the rdf:type property.

\section{RDFS- Describing Properties}

RDF Schema allows us to describe properties. Properties are instances of the class rdf:Property. We specify a domain using rdfs:domain and a range by using rdfs:range. RDF Schema semantically extends RDF by providing a means to describe RDF Vocabularies.

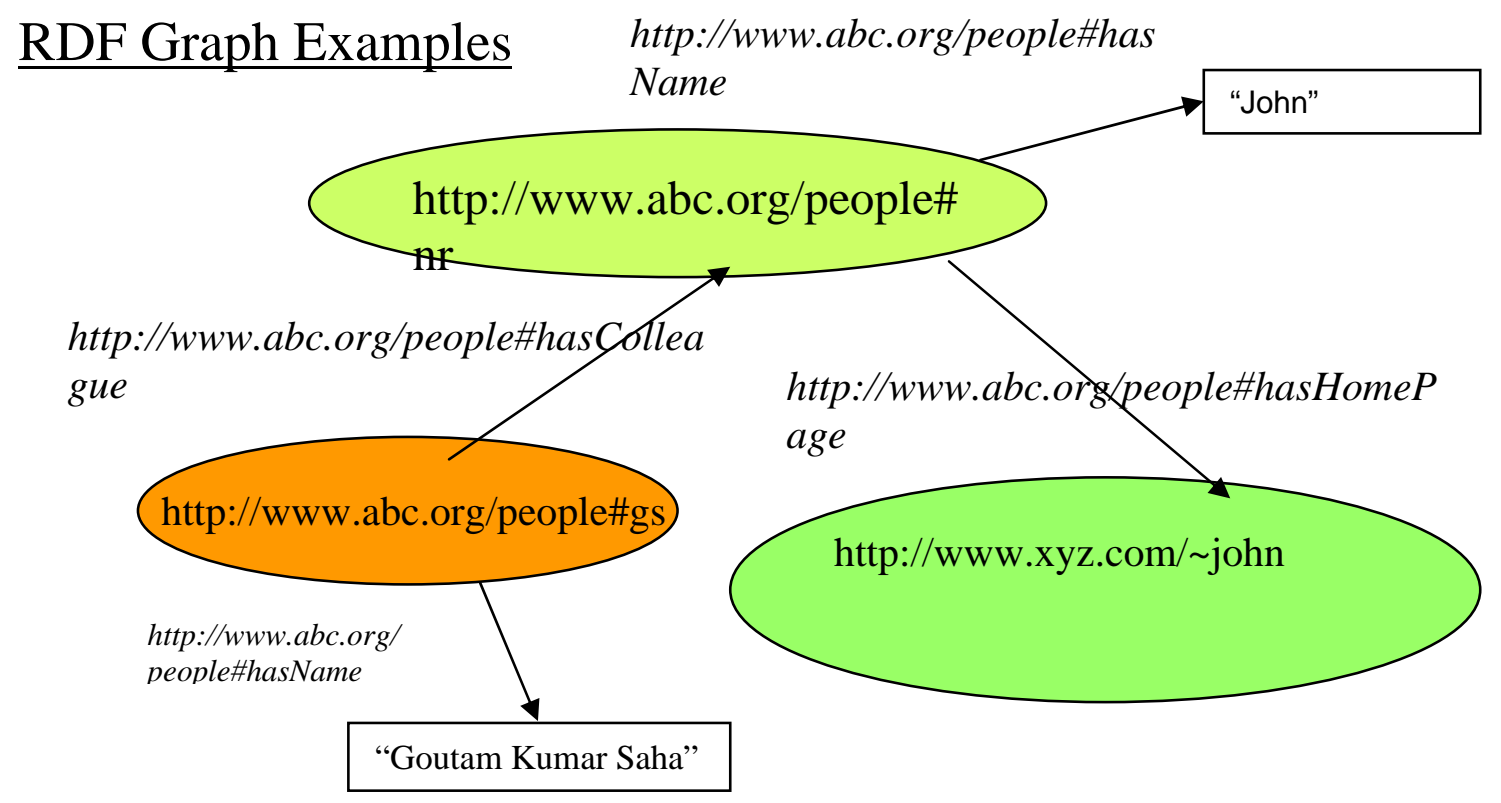




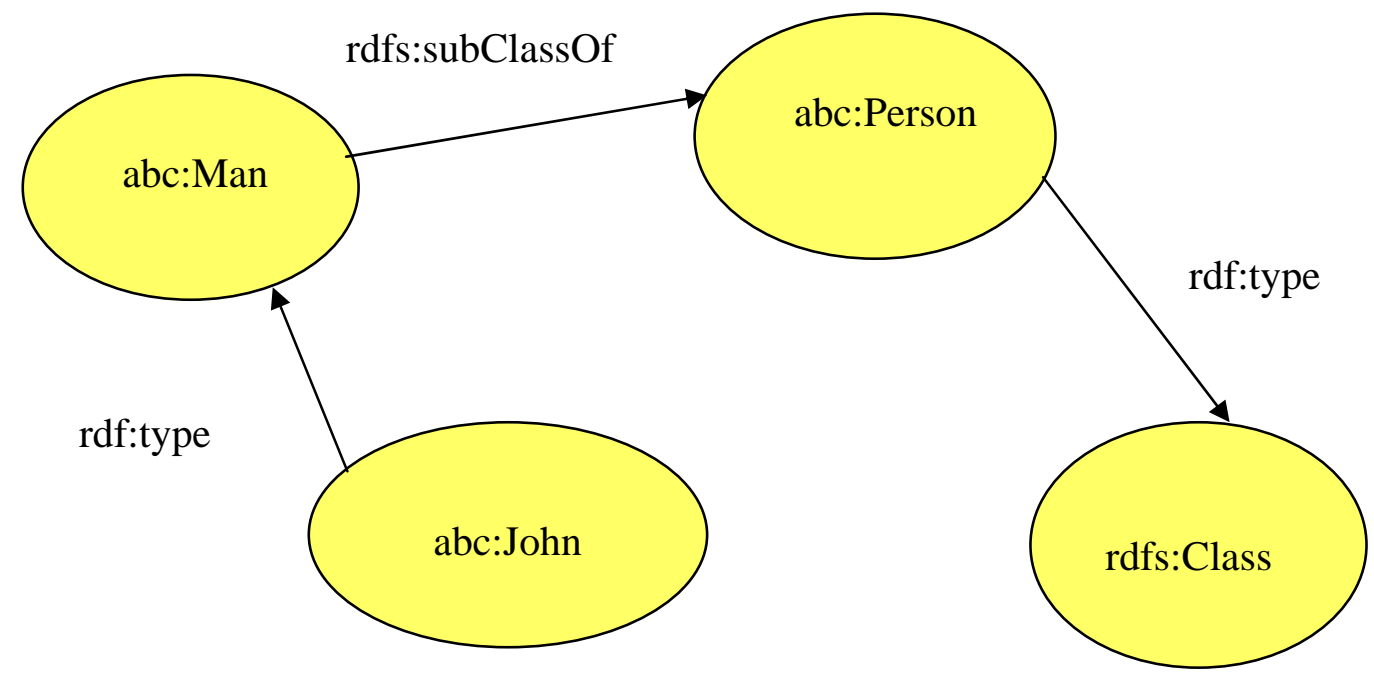

Ontology- a Layered Technology

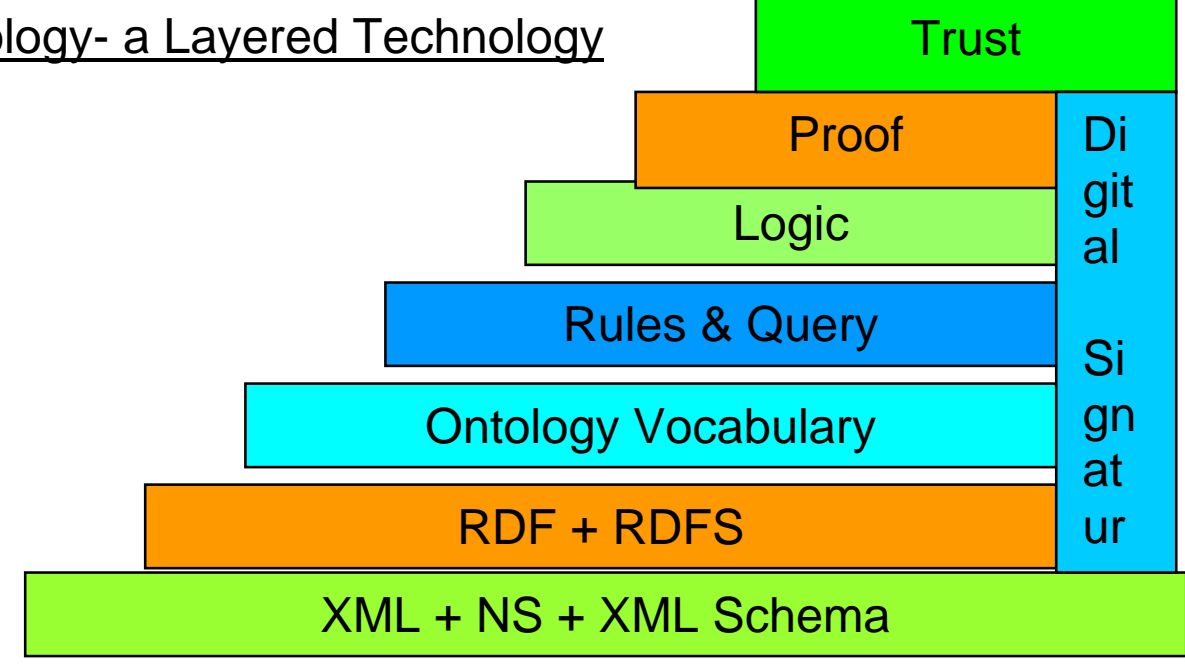

\begin{tabular}{|c|c|}
\hline Unicode & URI \\
\hline
\end{tabular}


$\underline{\text { RDFS- Describing Properties }}$

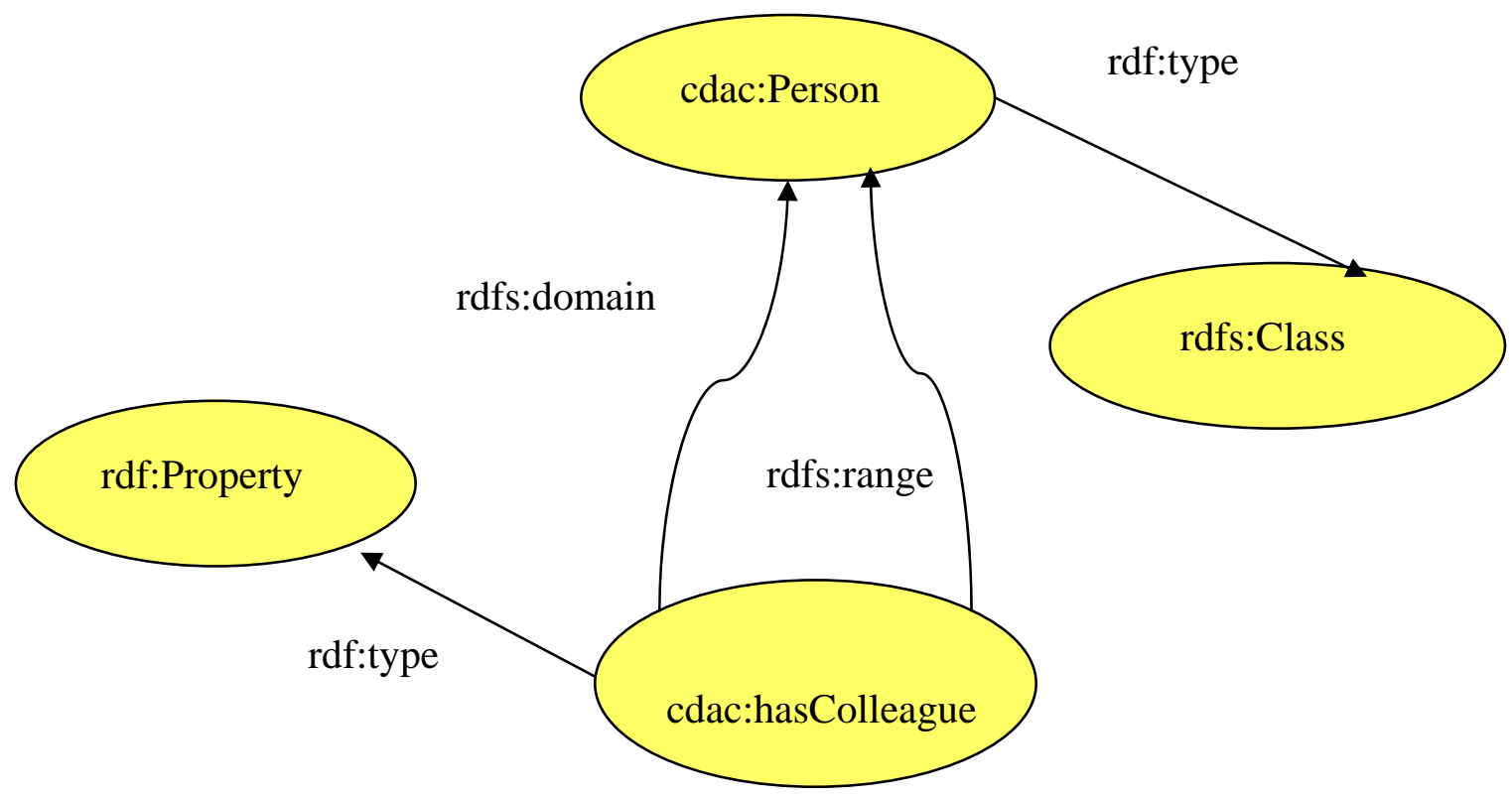

OWL: OWL Reasoning

For ontologies in OWL-DL, we can use an automatic reasoner to infer information that isn't explicitly represented in ontology.

Standard 'reasoning services' are Subsumption testing, Equivalence testing, Instantiation testing and Consistency testing.

\section{OWL Classes}

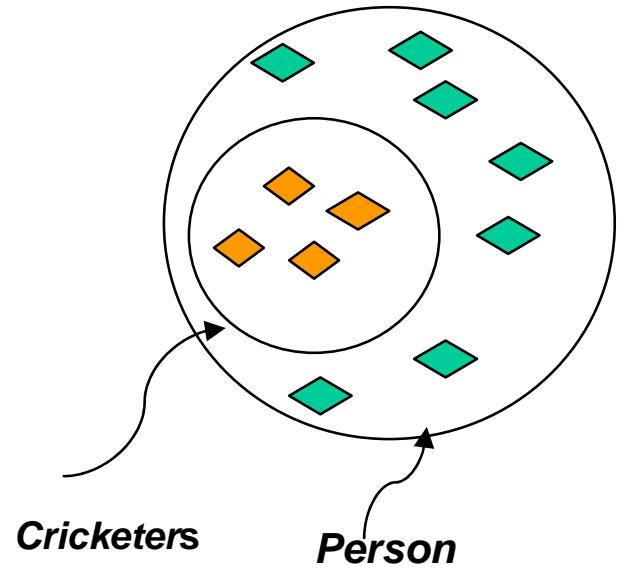

Named Classes
Intersection Classes:

$A \cap B=\{x \mid x \in A$ and $x \in B\}$

e.g. Engineer $\cap$ Male

\section{Union Classes:}

$A \cup B=\{x \mid x \in A$ or $x \in B\}$

e.g. Doctor UEngineer 
Existential Restriction ( $\exists$ at least one)

$\exists \mathrm{RC}$ is interpreted as the set of all individuals in the domain which has at least one R-successor.

\section{Universal Restrictions $\forall \mathbf{R} \mathbf{C}$}

Value restriction $\forall \mathrm{R} \mathrm{C}$ is interpreted as the set of all individuals in the domain whose all R-successors (if any) belong to the interpretation of concept or class C.

Complement Classes: Professional $\cap \neg$ Doctor

\section{Existential Restrictions}

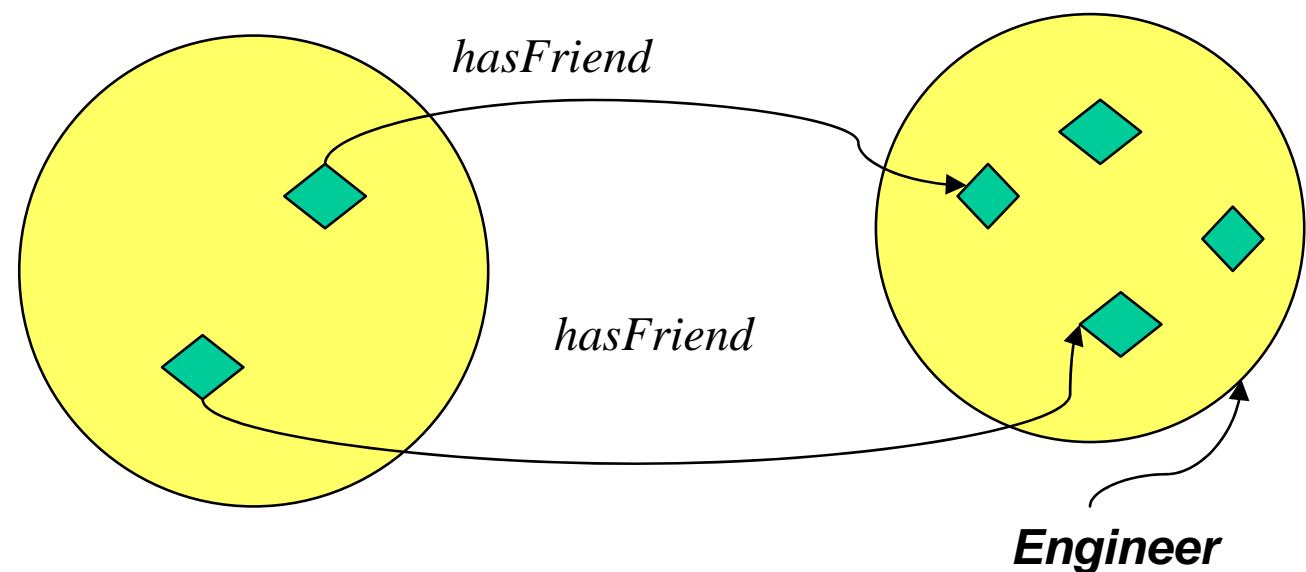

$\exists$ hasFriend Engineer

\section{Universal Restrictions hasFriend}

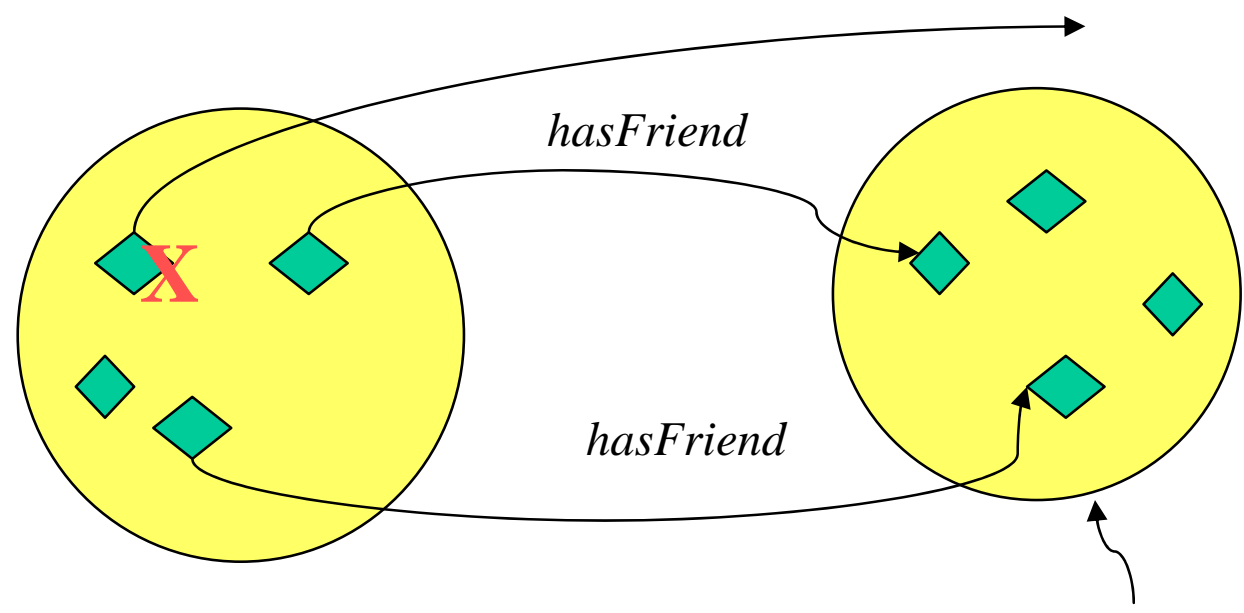

$\forall$ hasFirend Doctor

Doctor 


\section{Enumeration Classes}

An enumeration class is specified by explicitly and exhaustively listing the individuals that are members of the enumeration class. In order to specify an enumerated class, the individuals that are members of the class are listed inside curly brackets $\{\ldots \ldots\}$

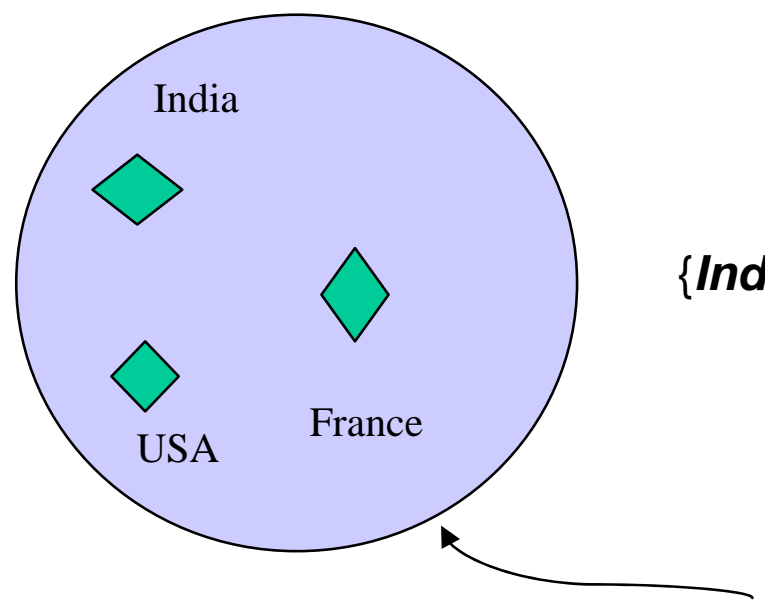

\{India USA France $\}$

\section{TouristDestination}

\section{OWL Coding Examples}

\section{OWL- Named Classes}

<owl:Class rdf:ID="Engineer">

$<$ rdfs:subClassOf rdf:resource="\#Professional" /> ...

$<$ lowl:Class $>$

\section{OWL- Enumerated Classes}

<owl:Class rdf:ID="InkColor">

$<$ rdfs:subClassOf rdf:resource="\#InkDescription" />

<owl: oneof rdf:parseType="Collection">

<owl:Thing rdf:about="\#Black"/>

<owl:Thing rdf:about="\#Blue"/>

$<$ lowl:oneOf $>$

<owl:Thing rdf:about="\#Red"/>

$<$ lowl:Class $>$

\section{OWL- Complement Classes}

<owl:Class rdf:ID="ConsumableThing" />

$<$ owl:Class rdf:ID="NonConsumableThing">

$<$ owl:complementOf rdf:resource="\#ConsumableThing" />

$</$ owl:Class $>$

\section{OWL- Union Classes}

<owl:Class rdf:ID="Fruit">

<owl:unionOf rdf:parseType="Collection"> 
<owl:Class rdf:about="\#SweetFruit" />

<owl:Class rdf:about="\#NonSweetFruit" />

$<$ /owl:unionOf>

$<$ lowl:Class $>$

\section{OWL- Intersection Classes \\ <owl:Class rdf:ID="LadyDoctor"> \\ <owl:intersectionof rdf:parseType="Collection"> \\ <owl:Class rdf:about="\#Doctor" /> \\ $<$ owl:Class rdf:about="\#Woman" /> \\ $</$ owl:intersectionOf $>$ \\ $<$ lowl:Class $>$}

\section{$\underline{\text { Restrictions }}$}

Cardinality Restrictions: For a given property, cardinality restrictions allow us to talk about the number of relationships that a class of individuals participates in.

hasValue Restrictions: - Allow us to specify that class of individuals that participate in a specified relationship with a specific individual.

\section{hasValue Restriction}

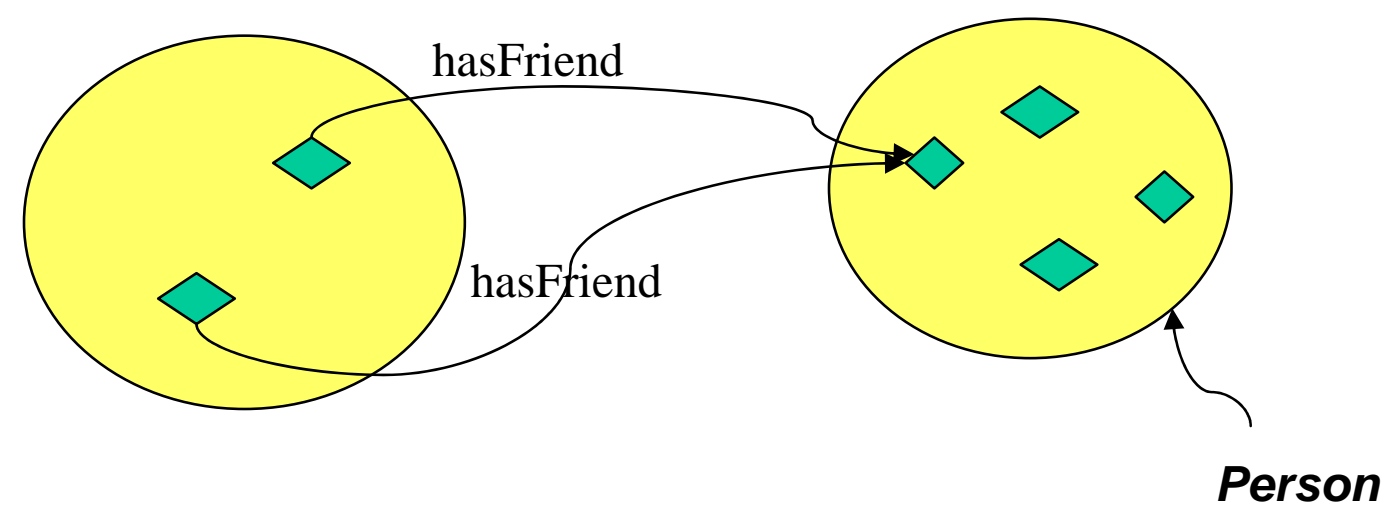

\section{hasFirend ${ }_{\ni}$ Ram}

\section{$\underline{\text { OWL Properties }}$}

There are two main categories of properties: Object properties and datatype properties.

Object properties link individuals to individuals. Datatype properties link individuals to datatype values (e.g. integers, floats, strings).

Properties can have specified domain and range. Object properties my have an inverse property e.g. the inverse of hasFriend might be hasEnemy. 


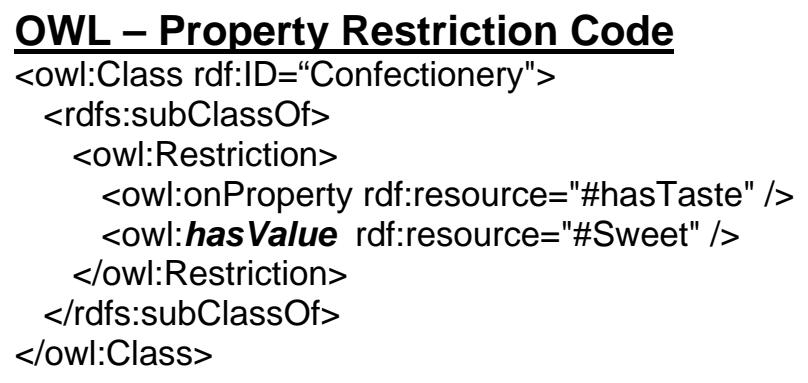

\section{Summary}

$\mathrm{OWL}$ is the latest standard in ontology languages (W3C Recommendation February 2004). It is layered on top of RDF and RDFS, and has a rich set of constructs. There are three categories of OWL: OWL-Lite, OWL-DL and OWLFull. Automated reasoning is possible over ontologies that are written in OWLLite and OWL-DL. OWL brings relationships to RDF. OWL allows individual instances of classes to be included in the ontology. OWL classes and individuals can be stored as RDF triples. RDF extends the XML Model and Syntax to be specified for describing resources. OWL assigns a specific meaning to RDF Triples. OWL is derived from the DAML + OIL Web Ontology Language and builds upon the RDF. OWL ontology language differs from an XML schema in that it is meant for knowledge representation, not for a message formatting. XML and RDF formats are part of the OWL standard. OWL is useful for various applications such as knowledge representation, knowledge management, photo retrieval, access control policies and so on. We may use further rules extension to OWL i.e. SWRL (Semantic Web Rule Language).

\section{Author's Biography}

In his last nineteen and half years' R\&D and teaching experience, Goutam Kumar Saha has worked as a scientist in LRDE, Defence Research \& Development Organisation, Bangalore and at the Electronics Research \& Development Centre of India, Calcutta. At present, he is with the Centre for Development of Advanced Computing, Kolkata, India, as a Scientist-F. He is a fellow in IETE and senior member in IEEE, Computer Society of India, and ACM etc. He has received various awards, scholarships and grants from national and international organizations. He is a referee of CSI Journal, AMSE Journal (France), IJCPOL (USA), IJCIS (Canada) and of an IEEE Journal / Magazine (USA). He is an associate editor of the ACM Ubiquity (USA), International Journal of the Latin American Center for Informatics Studies (CLEIJ) and of the International Journal of Computing and Information Sciences (Canada). He is a Chair and Member of the Program Committee of the WSNEXT / UBICOMM 2007 (French Polynesia), UBICOMM 2008 (France), IEEE Computer Society Press, CONFENIS2007 (Beijing) etc. His fields of interest include software based fault tolerance, web technology, EIS, Ontology Engineering and Natural Language 
Processing. He can be reached via sahagk@gmail.com, gksaha@ rediffmail.com.

\section{Author acknowledges various important Web Resources as References to this paper including:-http://www.w3.org/2004/OWL/; $R$. Studer;} http://www.w3.org/TR/webont-req/; http://www.w3.org/TR/owl-features; J. Kelly, The Essence of Logic, Prentice Hall, 1997; OWL Foundations, University of Manchester; Ayesha Kaljuvee, XML, Ontologies, and the Semantic Web, XML Journal, 2003; Protégé OWL Web Site; CO-ODE Web Site; Tim Berners-Lee, James Hendler, and Ora Lassila, The Semantic Web, Scientific American, 2001; and others.

\section{OWL Code Example for Web Resource Access Control Policies:}

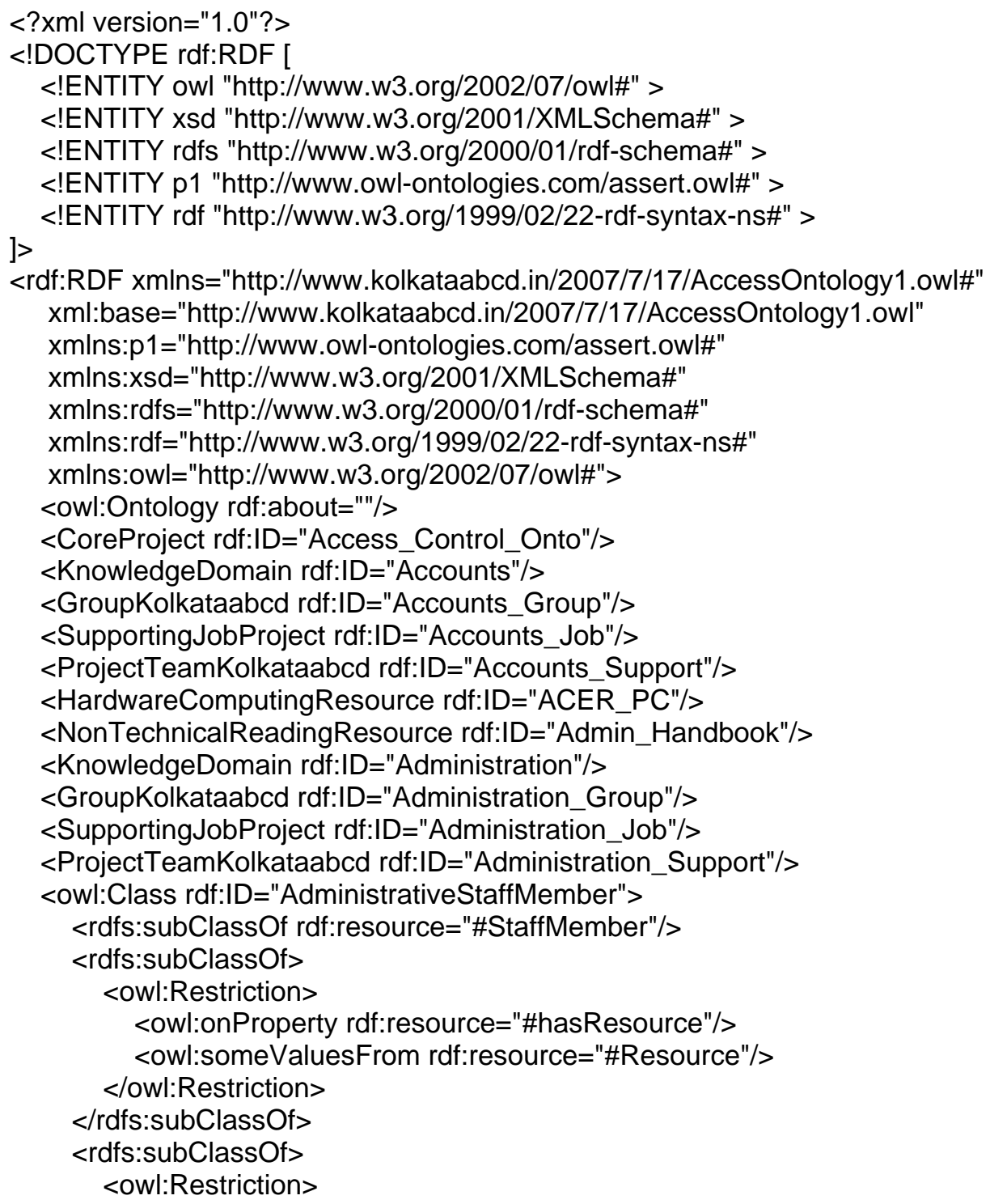




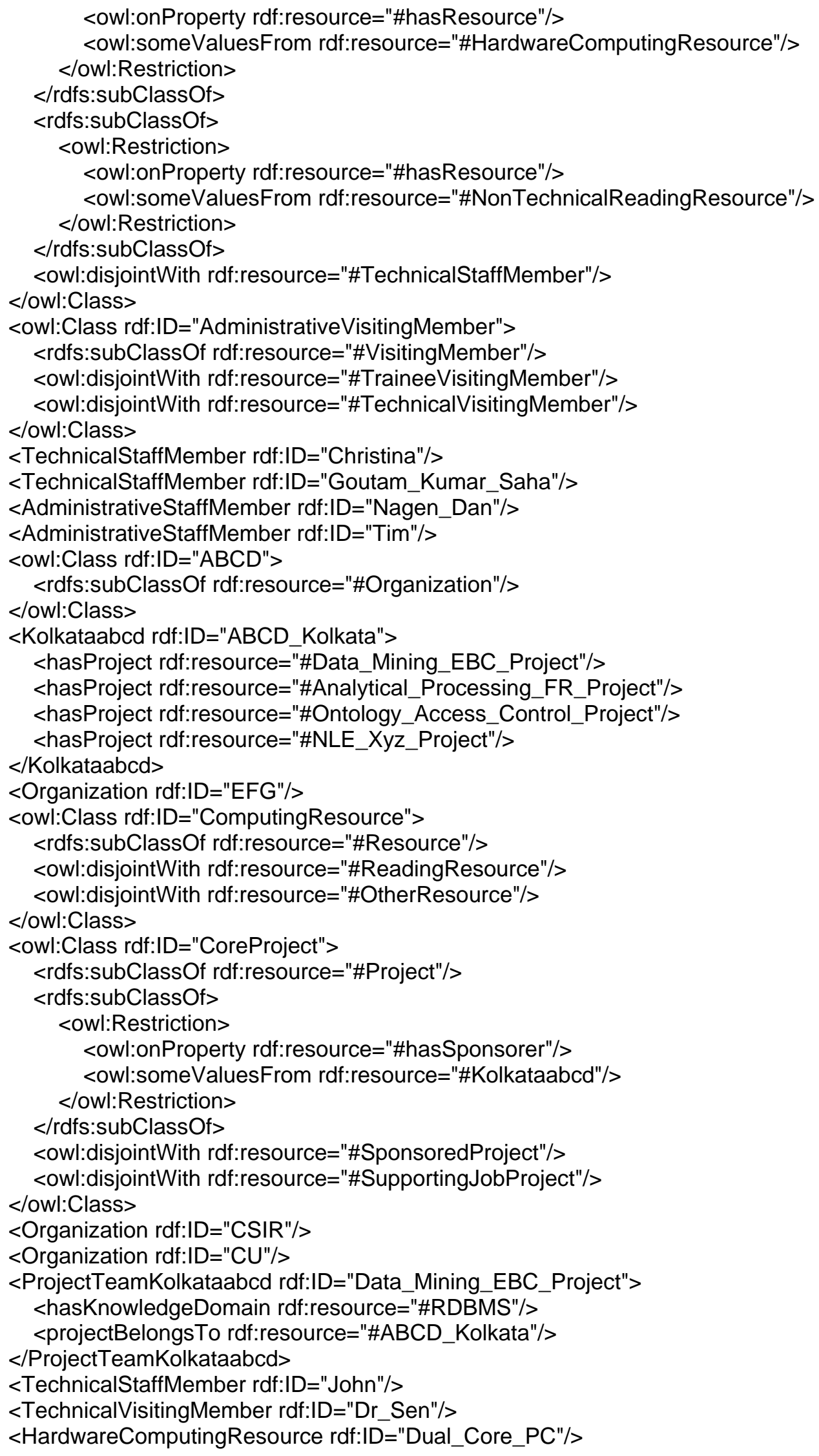




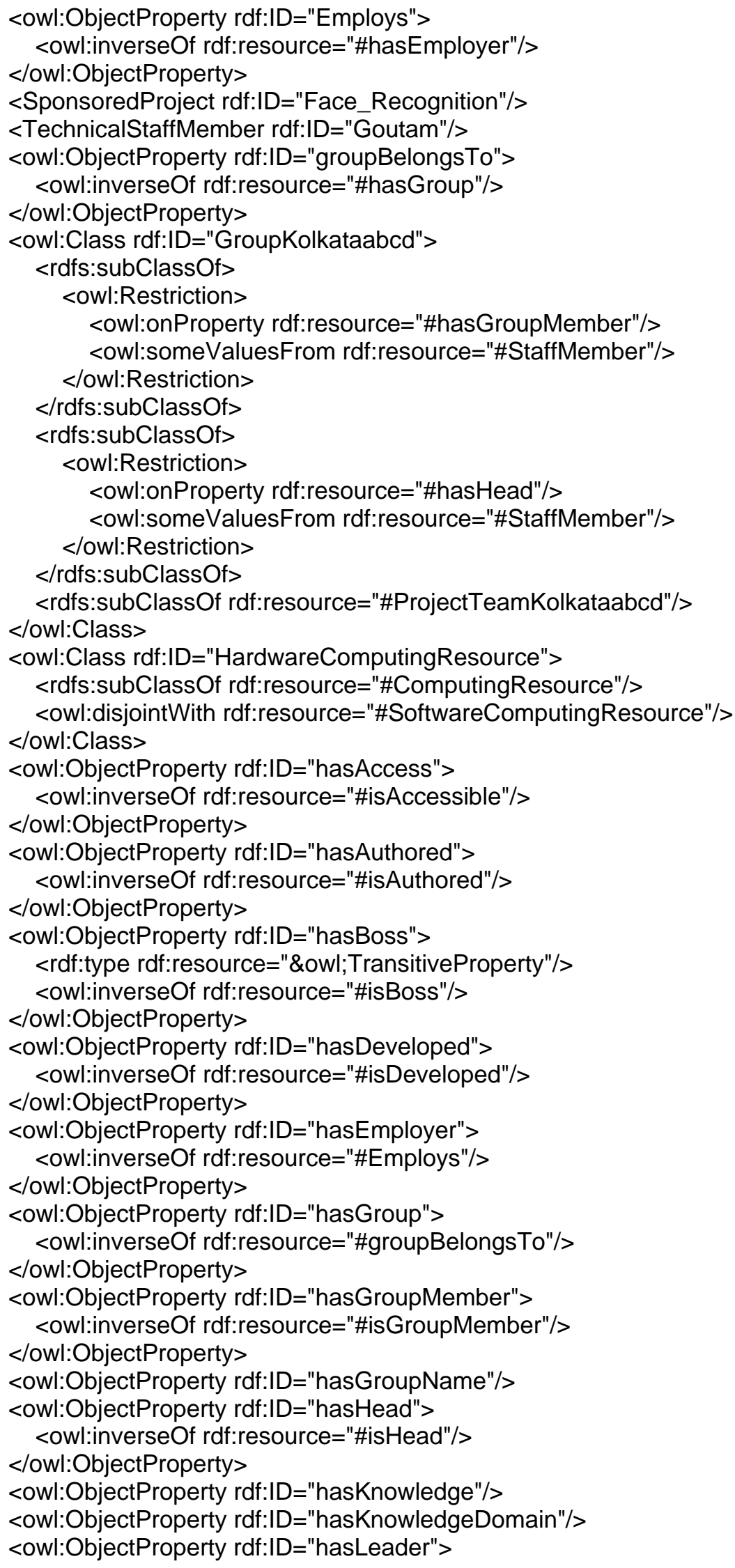




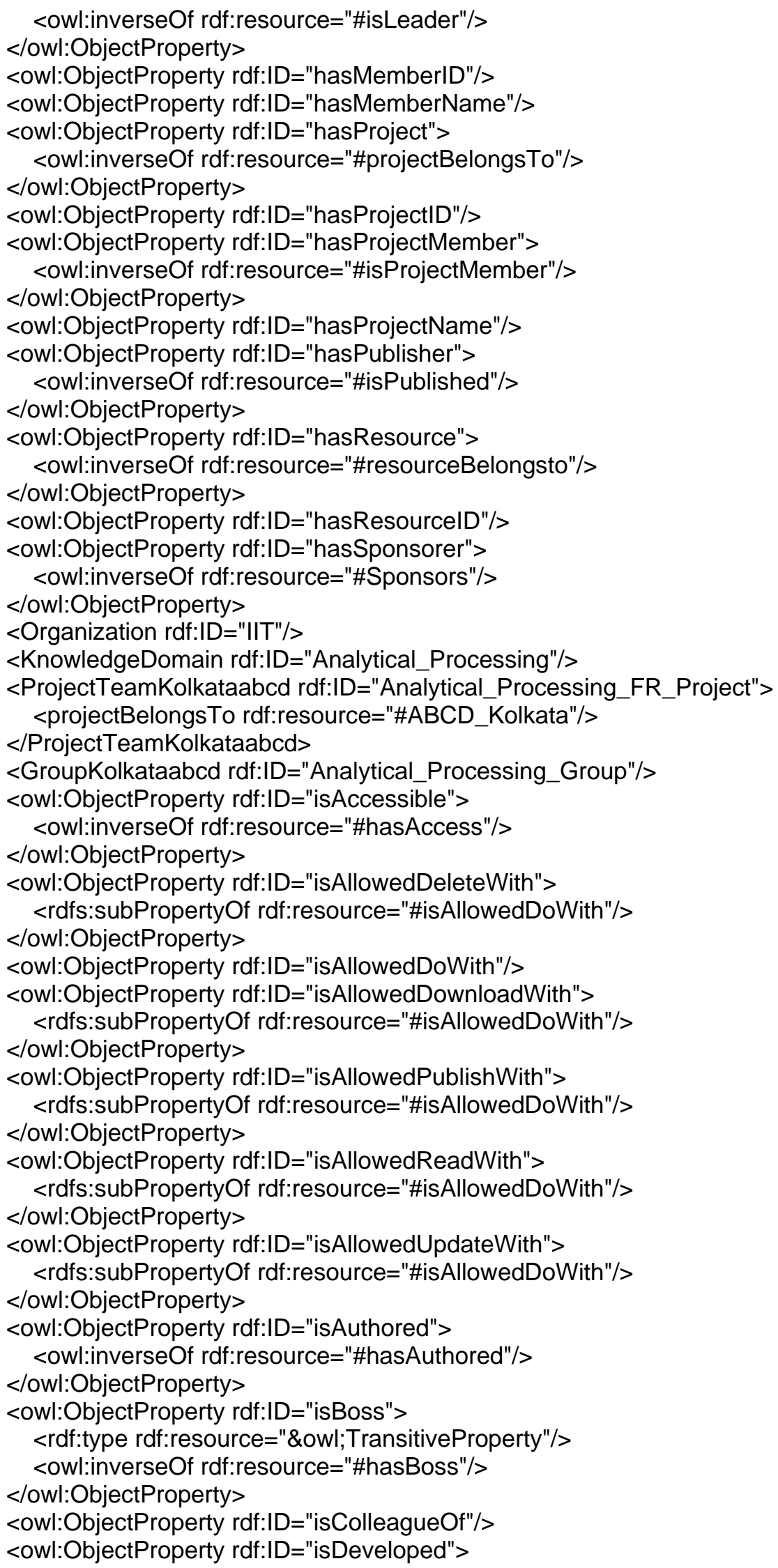


<owl:inverseOf rdf:resource="\#hasDeveloped"/>

$<$ /owl:ObjectProperty $>$

<owl:ObjectProperty rdf:ID="isGroupMember">

<owl:inverseOf rdf:resource="\#hasGroupMember"/>

$<$ /owl:ObjectProperty>

<owl:ObjectProperty rdf:ID="isHead">

<owl:inverseOf rdf:resource="\#hasHead"/>

$</$ owl:ObjectProperty>

<owl:ObjectProperty rdf:ID="islgnorant"/>

<owl:ObjectProperty rdf:ID="isLeader">

<owl:inverseOf rdf:resource="\#hasLeader"/>

$<$ /owl:ObjectProperty $>$

<owl:ObjectProperty rdf:ID="isNotAllowedDeleteWith">

$<$ rdfs:subPropertyOf rdf:resource="\#isNotAllowedToDoWith"/>

$</$ owl:ObjectProperty $>$

<owl:ObjectProperty rdf:ID="isNotAllowedDownloadWith">

$<$ rdfs:subPropertyOf rdf:resource="\#isNotAllowedToDoWith"/>

$<$ owl:ObjectProperty $>$

<owl:ObjectProperty rdf:ID="isNotAllowedPublishWith">

$<$ rdfs:subPropertyOf rdf:resource="\#isNotAllowedToDoWith"/>

$<$ /owl:ObjectProperty $>$

<owl:ObjectProperty rdf:ID="isNotAllowedReadWith">

<rdfs:subPropertyOf rdf:resource="\#isNotAllowedToDoWith"/>

$<$ owl:ObjectProperty $>$

<owl:ObjectProperty rdf:ID="isNotAllowedToDoWith"/>

<owl:ObjectProperty rdf:ID="isNotAllowedUpdateWith">

$<$ rdfs:subPropertyOf rdf:resource="\#isNotAllowedToDoWith"/>

$<$ /owl:ObjectProperty $>$

<owl:ObjectProperty rdf:ID="isProjectMember">

<owl:inverseOf rdf:resource="\#hasProjectMember"/>

$<$ /owl:ObjectProperty $>$

<owl:ObjectProperty rdf:ID="isPublished">

<owl:inverseOf rdf:resource="\#hasPublisher"/>

$<$ /owl:ObjectProperty $>$

<owl:ObjectProperty rdf:ID="isSameGroupOf"/>

<owl:ObjectProperty rdf:ID="isSameProjectMemberOf">

< rdf:type rdf:resource="\&owl;TransitiveProperty"/>

$<$ /owl:ObjectProperty $>$

<owl:ObjectProperty rdf:ID="isSisterUnit">

<rdf:type rdf:resource="\&owl;TransitiveProperty"/>

$<$ /owl:ObjectProperty>

<owl:ObjectProperty rdf:ID="isStrong">

<owl:inverseOf rdf:resource="\#isWeak"/>

$<$ /owl:ObjectProperty $>$

<owl:ObjectProperty rdf:ID="isWeak">

<owl:inverseOf rdf:resource="\#isStrong"/>

$<$ /owl:ObjectProperty>

$<$ SoftwareComputingResource rdf:ID="Java"/>

$<$ TechnicalStaffMember rdf:ID="JTimta"/>

$<$ Organization rdf:ID="JU"/>

<owl:Class rdf:ID="KnowledgeDomain">

<owl:disjointWith rdf:resource="\#Person"/>

$<$ owl:disjointWith rdf:resource="\#Project"/>

<owl:disjointWith rdf:resource="\#Organization"/>

<owl:disjointWith rdf:resource="\#Resource"/>

$</ o w l: C l a s s>$ 


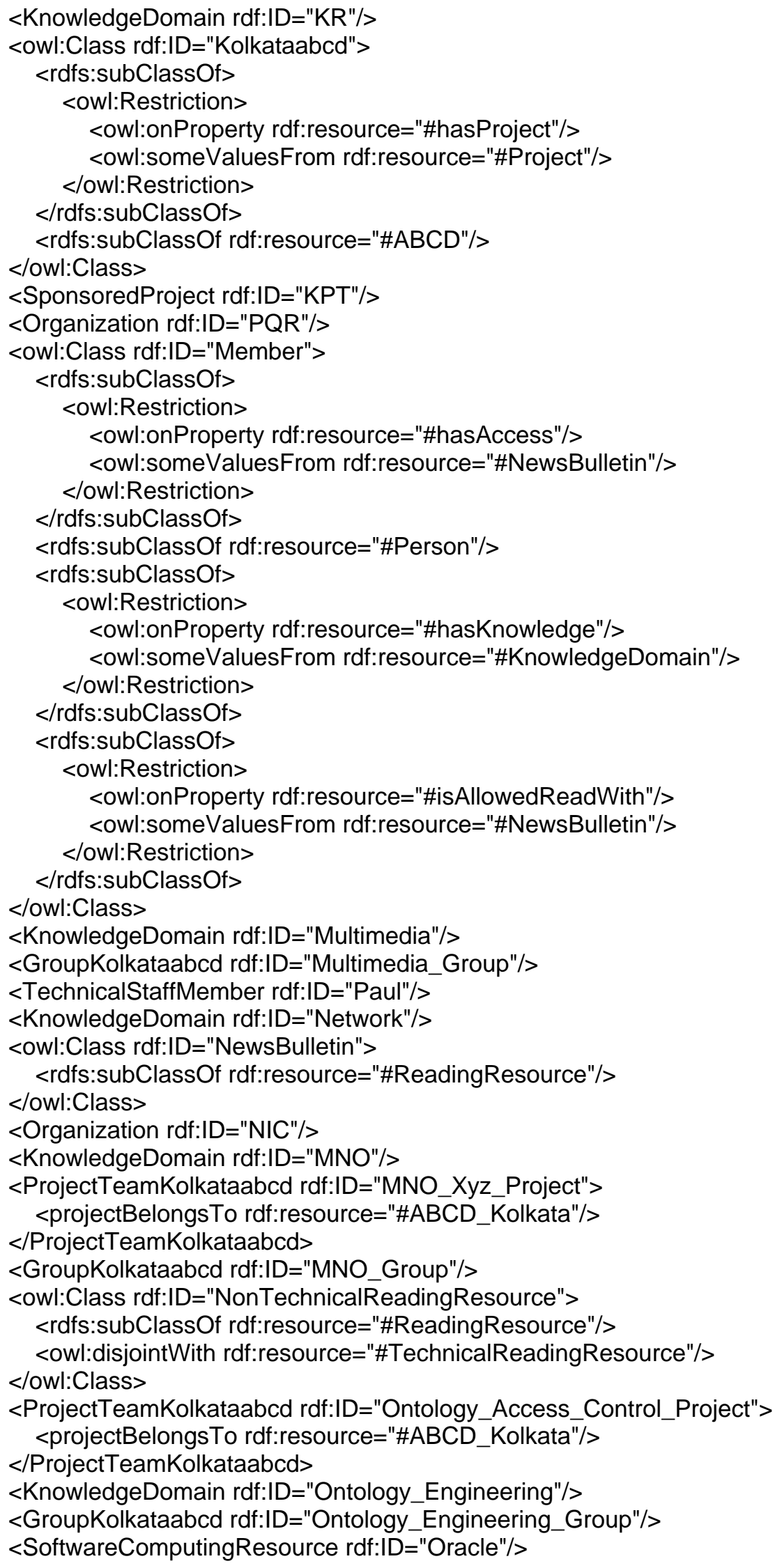




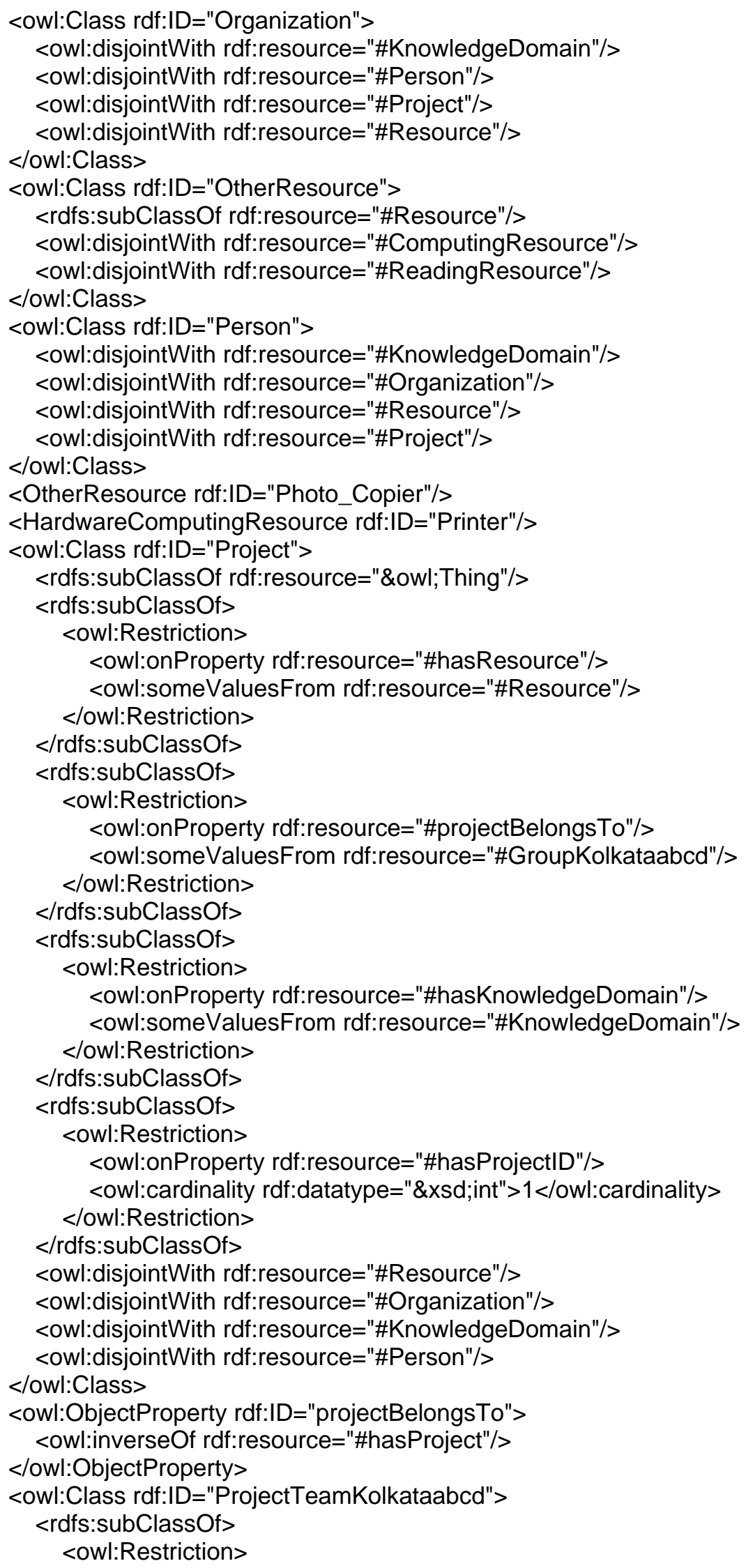




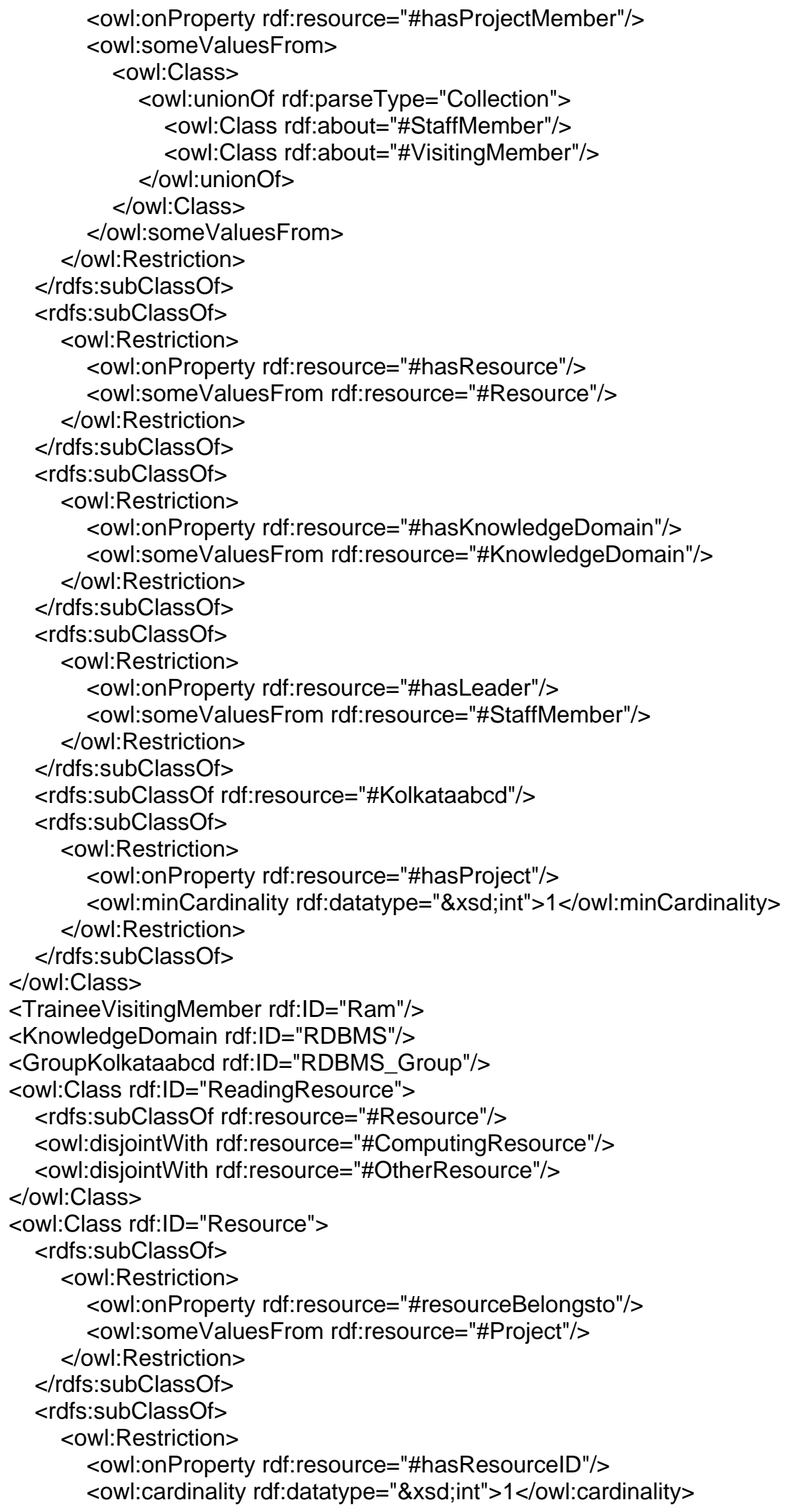




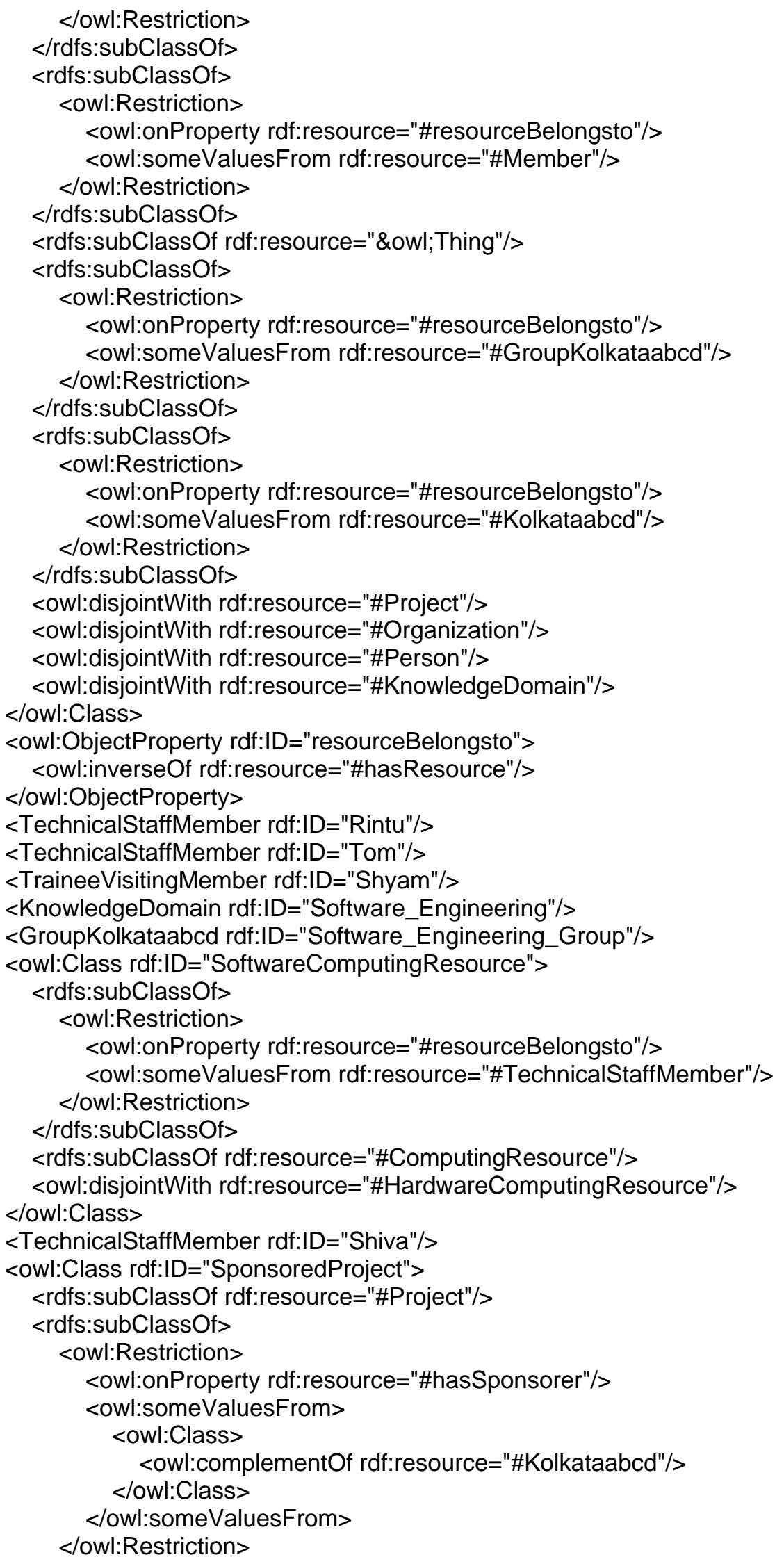




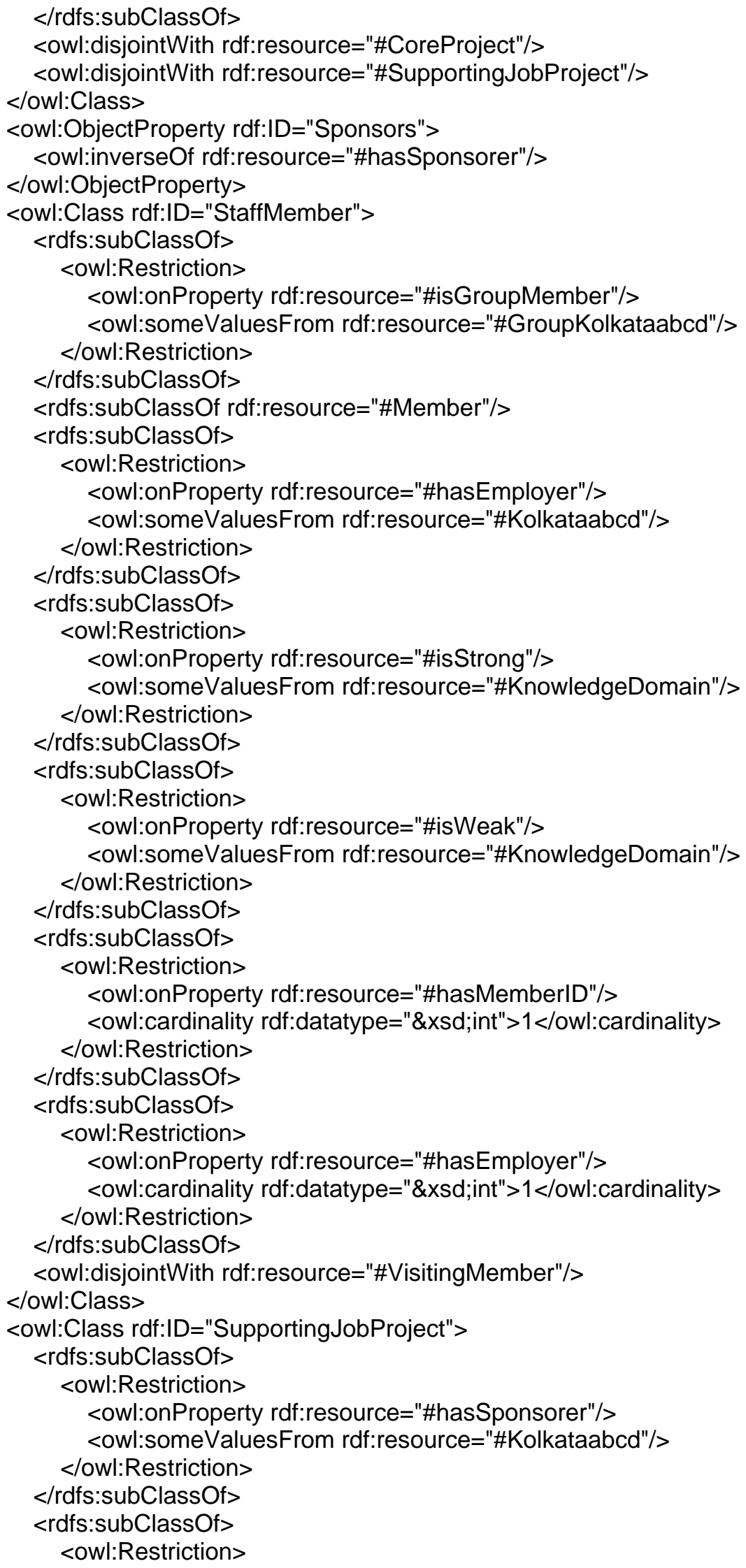




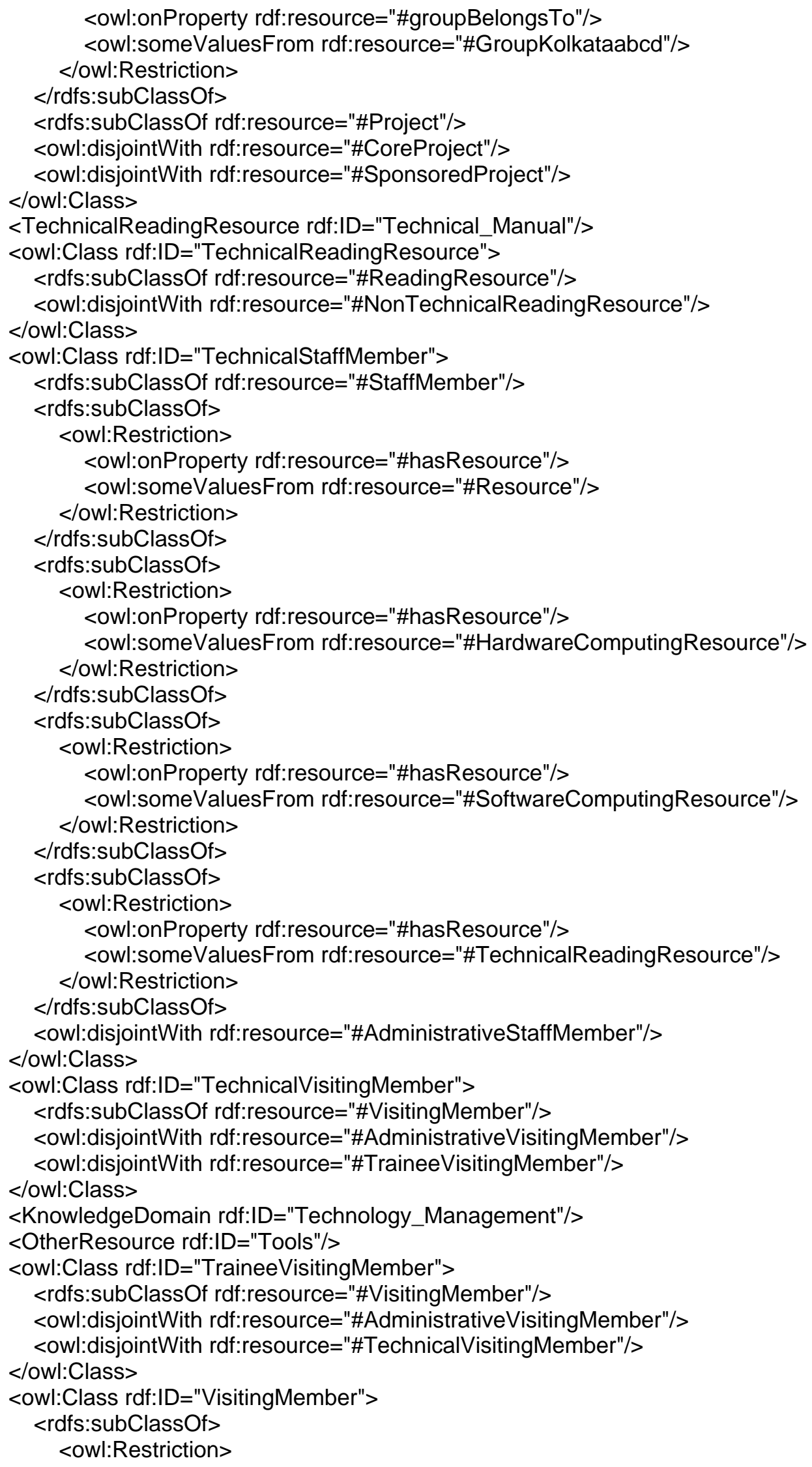




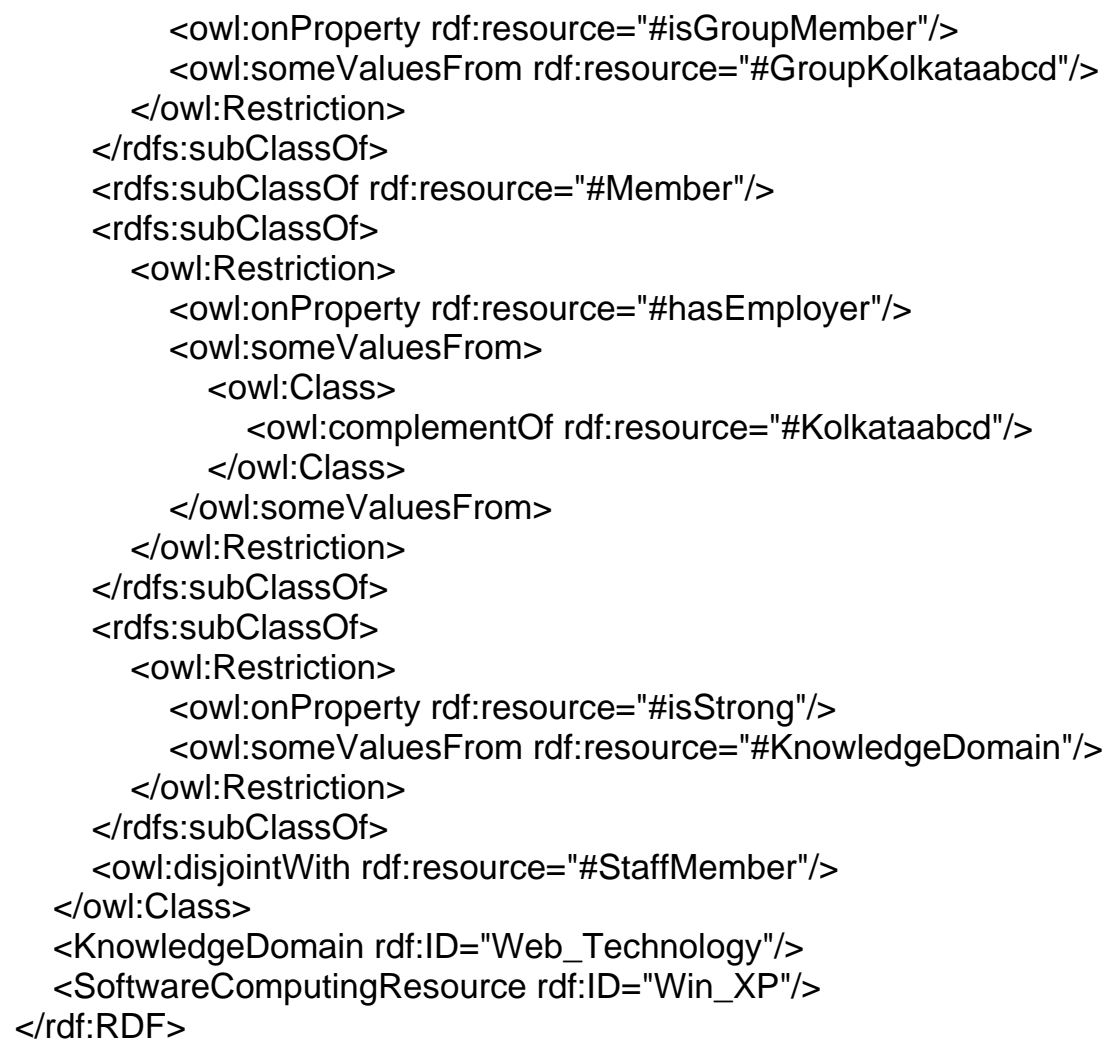

